\title{
O RECONTO DE HISTÓRIAS EM CRIANÇAS DO ESPECTRO AUTÍSTICO: UM ESTUDO PRELIMINAR
}

\section{Retelling a story in autistic spectrum children: a preliminary study}

\author{
Juliana Onofre de Lira ${ }^{(1)}$, Ana Carina Tamanaha ${ }^{(2)}$, Jacy Perissinoto ${ }^{(3)}$, Ellen Osborn ${ }^{(4)}$
}

\begin{abstract}
RESUMO
Objetivo: verificar a habilidade de crianças diagnosticadas como autistas ou com Síndrome de Asperger em recontar uma história. Métodos: foram avaliados quatro meninos verbais, com idade entre cinco e dez anos, diagnosticados por equipe multidisciplinar com Autismo Infantil (2) e Síndrome de Asperger (2) e atendidos no Laboratório de Investigação Fonoaudiológica - Transtornos Globais do Desenvolvimento - Departamento de Fonoaudiologia da Universidade Federal de São Paulo (UNIFESP). Foi utilizada a história "Urubu e as pombas", proposta por Golden revisada e adaptada por Antunes e col, que deveria ser reproduzida após a narrativa realizada pela avaliadora. A análise do desempenho lingüístico foi dividida em quantitativa através do número total de sentenças (nodos) reproduzidas (14 no total), e qualitativa pelo reconto dos temas centrais da história e a ocorrência de distorções (as alterações que podem ocorrer no reconto da história). Resultados: foi verificado que o número de sentenças reproduzidas foi sete, em média. No entanto, é interessante notar que nem todos os nodos considerados tema central foram recontados. Conclusão: os resultados sugerem que a habilidade de memória textual evidenciada nos índices de reprodução das orações não foi suficiente para garantir compreensão da história, dada ausência de alguns nodos dos temas centrais no reconto realizado pelos sujeitos.
\end{abstract}

DESCRITORES: Transtorno Autístico; Síndrome de Asperger; Memória; Compreensão

(1) Fonoaudióloga; Membro do Grupo de Pesquisa em Transtornos de Linguagem do Conselho Nacional de Desenvolvimento Científico e Tecnológico, GPTL - CNPq; Mestranda em Distúrbios da Comunicação Humana pela Universidade Federal de São Paulo.

(2) Fonoaudióloga; Professora Doutora Convidada do Departamento de Fonoaudiologia da Universidade Federal de São Paulo, UNIFESP, São Paulo, SP; Membro do Grupo de Pesquisa em Transtornos de Linguagem do Conselho Nacional de Desenvolvimento Científico e Tecnológico, GPTL - CNPq e Supervisora do Laboratório de Investigação Fonoaudiológica de Linguagem nos Transtornos Globais do Desenvolvimento do Departamento de Fonoaudiologia da Universidade Federal de São Paulo; Doutora em Distúrbios da Comunicação Humana pela Universidade Federal de São Paulo.

(3) Fonoaudióloga; Professora Associada do Departamento de Fonoaudiologia da Universidade Federal de São Paulo, UNIFESP, São Paulo, SP; Coordenadora do Grupo de Pesquisa em Transtornos de Linguagem do Conselho Nacional de Desenvolvimento Científico e Tecnológico, GPTL - CNPq e do Laboratório de Investigação Fonoaudiológica - Transtornos Globais do Desenvolvimento do Departamento de Fonoaudiologia da Universidade Federal de São Paulo; Doutora em Distúrbios da Comunicação Humana pela Universidade Federal de São Paulo.

(4) Fonoaudióloga; Professora Adjunto do Departamento de Fonoaudiologia da Universidade Federal de São Paulo, UNIFESP, São Paulo, SP; Mestre em Distúrbios da Comunicação Humana.

Este artigo é dedicado à memória da Prof. ${ }^{a}$ Dr. ${ }^{\text {a }}$ Márcia Regina Marcondes Pedromônico.

Conflito de Interesses: Inexistente

\section{INTRODUÇÃO}

Os quadros que compõem o espectro autístico são caracterizados por prejuízos em diversas áreas do desenvolvimento, entre elas, a interação social recíproca, comunicação e interesses ${ }^{1,2}$. Dentre as diversas manifestações clínicas, os indivíduos acometidos por esses transtornos podem demonstrar capacidades cognitivas e lingüísticas atípicas e peculiares, tais como excelente memória para detalhes.

A memória pode ser vista como uma habilidade de estocar e recuperar estímulos ${ }^{3}$.

Existem vários estudos relacionados ao desempenho em tarefas de memória verbal de eventos recentes. Alguns autores relatam pouca evidência de prejuízo na memória de autistas de alto funcionamento. Outros estudos apontam déficits, especialmente em autistas com comprometimento intelectual ${ }^{4-7}$.

Em indivíduos com Síndrome de Asperger, que também compõem o espectro autístico, há estudos que mostram desempenho adequado na memória verbal de eventos recentes ${ }^{5}$. No entanto, outras 
pesquisas demonstram haver prejuízo na recordação de estímulos verbais recentes a partir de categorias semânticas. Quando estes mesmos indivíduos são solicitados a recontar eventos recentes não relacionados entre si, o desempenho ocorre de forma adequada ${ }^{8}$.

Diferentes autores também encontraram sujeitos pertencentes ao espectro autístico que apresentavam em tarefas verbais, desempenho pior com estímulos que exigiam memória relacionada à organização semântica da informação ${ }^{9,10}$. Segundo o último dos estudos citados, a justificativa para tal desempenho poderia ser a diferença na organização da memória semântica ou o prejuízo na codificação do material verbal.

Esse padrão de desempenho pode não ser resultado de um déficit de memória, mas de um prejuízo na aplicação de estratégias gerais, como por exemplo, a organização da informação dentro de categorias, para posterior lembrança.

O fato de indivíduos do espectro autístico não armazenarem informações de acordo com categorias semânticas está associado provavelmente, às falhas de raciocínio e de compreensão ${ }^{\text {4-10. }}$.

$O$ déficit de linguagem apresentado por autistas, principalmente no que diz respeito às alterações semânticas, ocasiona um prejuízo na compreensão e elaboração do discurso. Esses sujeitos têm uma grande dificuldade em formar conceitos abrangentes, advindos da abstração de traços relevantes de objetos e situações, pois costumam apegar-se aos aspectos irrelevantes, não conseguindo, assim, ter um entendimento global da situação ou do objeto ${ }^{8-10}$.

A partir destes referenciais teóricos e experiência clínica, há a hipótese de que indivíduos que fazem parte do espectro autístico têm alteração de compreensão em relação a fatos ou situações, ainda que os memorizem isoladamente.

Desta forma, o presente estudo tem como objetivo verificar a habilidade de crianças diagnosticadas como autistas ou com Síndrome de Asperger em recontar uma história.

\section{MÉTODOS}

Foram avaliados quatro meninos, verbais, na faixa etária de cinco a dez anos, diagnosticados por equipe multidisciplinar como portadores de Autismo Infantil (dois) e Síndrome de Asperger (dois). Estas crianças são acompanhadas no Laboratório de Investigação Fonoaudiológica - Transtornos Globais do Desenvolvimento - do Depto de Fonoaudiologia da Universidade Federal de São Paulo (UNIFESP).
Utilizou-se a história "O urubu e as pombas", que se constitui um dos itens da Bateria de Avaliação Neuropsicológica Luria Nebrasca ${ }^{11}$ proposta por Golden (1987), traduzida para a Língua Portuguesa por Antunes e col (1991) e estudada por Moraes, Chiari e Perissinoto (2001) ${ }^{12}$.

É uma história sem ilustração, composta por 14 sentenças, as quais são chamadas de nodos. Estes estão distribuídos em 3 (três) episódios, considerando que cada episódio apresenta uma parte introdutória, o desenvolvimento e seu fim. Na Figura 1, é possível visualizar o conteúdo da história, bem como a distribuição mencionada acima.

Os nodos de número 3 (três), 4 (quatro), 11 (onze) e 14 (quatorze) são considerados os formantes do tema central da história, por caracterizarem ações importantes. Moraes, Chiari e Perissinoto ${ }^{12}$ ainda denominaram o nodo 4 (quatro) como o de maior impacto da história, caracterizando o objetivo do primeiro episódio.

Segundo Mandler e Johnson (1977) ${ }^{13}$, o nodo 2 (dois) que caracteriza o cenário, apresenta considerável importância, pois o seu reconto pode garantir compreensão do resto da história.

A avaliação foi individual. Foi realizada uma única narrativa da história por um fonoaudiólogo familiar à criança e, logo em seguida, foi solicitada a cada criança a reprodução oral da mesma. $O$ reconto foi gravado em fita cassete e transcrito, posteriormente, para protocolo específico.

Os resultados foram analisados por dois fonoaudiólogos para fidedignidade dos registros de forma: a) quantitativa, pelo registro do número total de nodos reproduzidos e b) qualitativa, pelo registro da ocorrência da recontagem dos nodos que caracterizam os temas centrais da história, bem como de distorções da história ${ }^{14}$. Foram consideradas distorções as seguintes situações:

- Omissão: não-recontagem de um ou mais nodos;

- Substituição: troca na recontagem do conteúdo total do nodo por alguma informação externa à história;

- Acréscimo: recontagem parcial ou total do nodo, com acréscimo de alguma informação externa à história;

- Inversão: troca temporal na recontagem do nodo;

- Repetição: recontagem repetida do nodo.

Os responsáveis pelos sujeitos autorizaram a sua participação neste estudo, o qual foi aprovado pelo Comitê de Ética em Pesquisa (CEP) da UNIFESP, sob a inscrição $n .{ }^{\circ}$ 1594/03.

Foi realizada uma análise descritiva dos dados, com apresentação dos números total de nodos e de cada item da análise qualitativa. 


\section{História: "O urubu e as pombas" \\ Golden (1987); revisão e adaptação de Antunes e col (1991).}

\section{Episódio 1:}

1. Evento iniciador: Um urubu ouviu dizer

2. Cenário: que na casa das pombas havia muita comida.

3. Reação: Ele se pintou de branco

4. Objetivo: e voou até a casa das pombas

5/6. Resultado: As pombas acharam que ele era uma delas

7. Final: e deixaram ele entrar.

\section{Episódio 2:}

8. Evento iniciador: Ele continuou a gritar como um urubu.

9. Reação: As pombas descobriram

10. Resultado: que ele era um urubu

11. Final: e o expulsaram.

\section{Episódio 3:}

12. Objetivo: Ele tentou se juntar novamente aos urubus

13. Resultado: mas estes não o reconheceram

14. Conclusão: e não o aceitaram.

\section{Figura 1 - História "O urubu e as pombas” com suas subdivisões: episódios e nodos}

\section{RESULTADOS}

\section{Análise Quantitativa}

Na Figura 3, é possível observar a ocorrência de recontagem dos nodos que compõem a história, em cada sujeito avaliado. Em média, foram recontados 7 nodos.

Os nodos assinalados correspondem às sentenças que compõem os temas centrais da história.

\section{Análise Qualitativa}

$\mathrm{Na}$ Figura 4, é possível visualizar a ocorrência de omissões produzidas pelas crianças avaliadas.

A Figura 5 mostra a ocorrência de substituições produzidas pelos sujeitos.

Não foi observada a ocorrência de distorções dos tipos inversão (troca temporal na recontagem do nodo) e repetição (recontagem repetida do nodo) nos recontos das crianças avaliadas.

Houve apenas uma situação de acréscimo, realizada pelo sujeito 1 , no nodo 12 ("...Ele tentou se juntar novamente aos urubus."). Esta criança recontou este nodo da seguinte maneira "Ele se juntou com a pomba e os dinossauros".

\section{DISCUSSÃO}

Em relação à análise quantitativa, verificou-se que, em média, dos 14 nodos que compõem a história, sete nodos foram recontados.
Os dois meninos com Síndrome de Asperger recontaram maior número de nodos, pois o sujeito 3 (8 anos) foi capaz de recontar nove nodos e o sujeito 4 (7 anos), oito nodos. Os dois autistas reproduziram cinco nodos cada (sujeito 1 - 9 anos; sujeito 2 -10 anos).

Dos estudos compilados, a maioria dos autores acredita que o prejuízo na memória é menos significativo em indivíduos com Síndrome de Asperger ${ }^{7-10}$.

Apesar de ter ocorrido um bom desempenho na recontagem dos nodos, isto provavelmente não pode garantir a compreensão da história, pois isto demandaria que o conteúdo da história fosse organizado semanticamente.

Em um estudo em crianças com desenvolvimento típico ${ }^{12}$, foram considerados fatores que influenciam diretamente a memorização e a evocação: a existência dos conteúdos de impacto, bem como das unidades centrais ou de alto nível de significado. Portanto, o reconto de nodos que caracterizam o tema central da história - 3 ("Ele se pintou de branco"), 4 (“...e voou até a casa das pombas"), 11 (“...e o expulsaram.”) e 14 ("...e não o aceitaram.") - pode garantir a compreensão do conteúdo da história.

É possível verificar através da Figura 3 que três sujeitos recontaram os nodos 3 ("Ele se pintou de branco"), 11 (“...e o expulsaram.”) e 14 (“...e não o aceitaram."). 


\section{UNIFESP

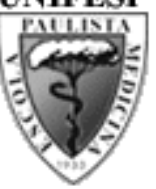

\section{UNIVERSIDADE FEDERAL DE SÃO PAULO}

Departamento de Fonoaudiologia

Disciplina dos Distúrbios da Comunicação Humana

Laboratório de Investigação Fonoaudiológica - Transtornos Globais do Desenvolvimento

Nome:

D.N: IA:

Data da Avaliação:

\section{ANÁLISE QUANTITATIVA \\ Recontagem}

1. Um urubu ouviu dizer/

2. Que na casa das pombas havia muita comida./

3. Ele se pintou de branco/

4. E voou até a casa das pombas./

5. As pombas acharam/

6. Que ele era uma delas/

7. E deixaram ele entrar,/

8. Mas ele continuou a gritar,/

9. Como um urubu./

10.As pombas descobriram que ele era um urubu/

11.E o expulsaram./

12.Ele tentou se juntar novamente aos urubus/

13.Mas estes não o reconheceram/

14.E não o aceitaram.

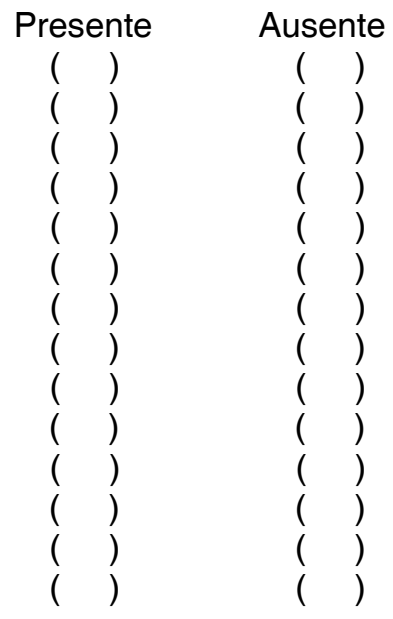

Itens contados corretamente: $/ 14$

\section{ANÁLISE QUALITATIVA}

( ) omissão do(s) nodo(s):

( ) substituição do(s) nodo(s):

( ) acréscimo de informação no(s) nodo(s):

( ) inversão do(s) nodo(s):

( ) repetição do(s) nodo(s):

Compreensão:
( ) Total
( ) Parcial
( ) Ausente

Baseado em MANDLER e JOHNSON (1977); GOLDEN(1987).

\section{Figura 2 - Protocolo utilizado na aplicação do procedimento}

No entanto, o mesmo não ocorreu com o nodo de número 4 ("..e voou até a casa das pombas"), que foi recontado apenas pelo sujeito 4 , criança com Síndrome de Asperger.

O nodo de número 4 (“..e voou até a casa das pombas") é denominado o de maior impacto da história ${ }^{12}$. Por este motivo, é possível correlacioná-lo, com maior ênfase, à compreensão da história.
Em relação à ocorrência de omissões no reconto (Figura 4), é possível verificar que todos os sujeitos avaliados omitiram os nodos de número 2 (dois) e 7 (sete).

O nodo 2 ("... que na casa das pombas havia muita comida.") explana o cenário da história "O urubu e as pombas". Há autores ${ }^{13}$ que destacaram a importância do reconto do cenário, pela relevân- 


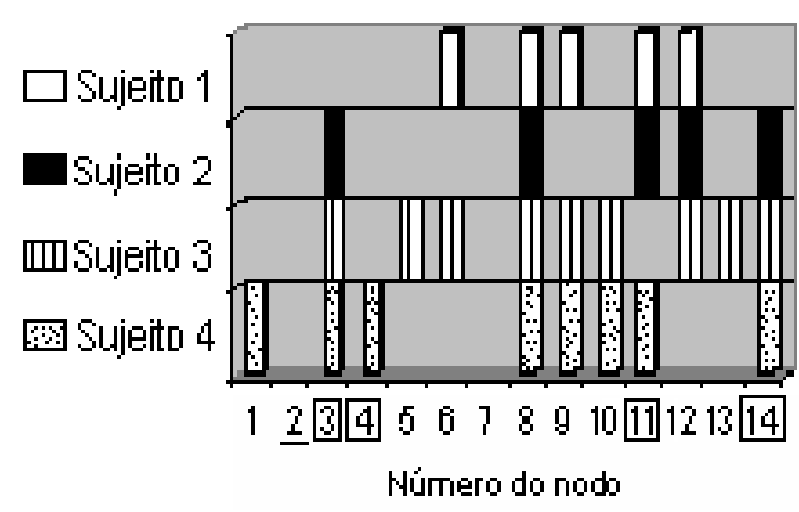

Figura 3 - Ocorrência de recontagem dos nodos pelos sujeitos avaliados

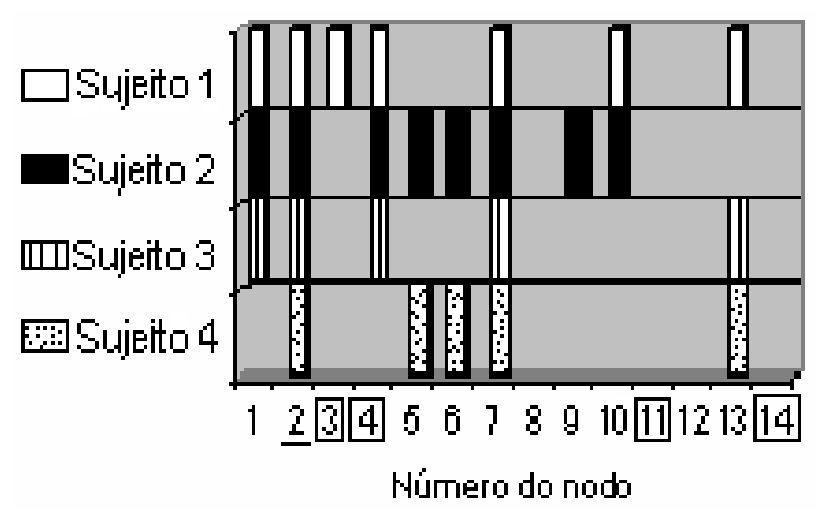

Figura 4 - Ocorrência de omissões dos nodos pelos sujeitos avaliados

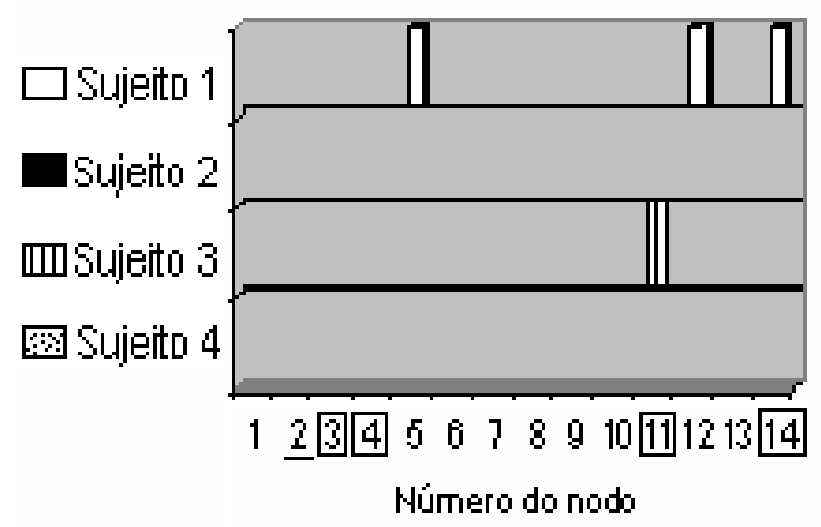

Figura 5 - Ocorrência de substituições dos nodos pelos sujeitos avaliados cia que este item apresenta para a compreensão do restante da narrativa.

$O$ fato de os quatro meninos apresentarem omissão desta parte da história no reconto reforça a hipótese da presença de déficits na compreensão da história utilizada.

Por outro lado, o alto índice de omissão do nodo 7 (“... as pombas deixaram ele entrar.") é concordante com os achados encontrados na literatura ${ }^{12}$ na avaliação de escolares sem queixa, de aproximadamente 9 (nove) anos de idade.

Houve um baixo índice de substituição no reconto e apenas os sujeitos 1 e 3 o fizeram (Figura 5). Apesar disto, a ocorrência de substituição dos nodos 11 (onze) e 14 (quatorze), os quais caracterizam o tema central da história, reforça a hipótese de déficit na compreensão da história.

Não foi observada a ocorrência de distorções dos tipos inversão e repetição. Houve apenas uma situação de acréscimo do sujeito 1 , no nodo 2 , lembrando que este nodo, que caracteriza o cenário, é fundamental para compreensão da narrativa ${ }^{13}$.

De modo geral, a ausência do reconto dos nodos 2 (cenário) e a reprodução do nodo 4 (tema central) por apenas uma criança reforça a hipótese de prejuízos no processamento e interpretação de informação provida de contexto e significado, o que pode acarretar déficits de compreensão desta informação ${ }^{7-10,14-17 .}$

Desta forma, há necessidade do prosseguimento deste estudo em uma amostra maior e no detalhamento sobre a compreensão das informações nos indivíduos estudados.

Para estudos posteriores acreditamos ser necessário o emprego de outras abordagens de apresentação de narrativas, como a utilização de figuras associadas ou sequências de figuras para auxiliar no estudo de memória e compreensão em indivíduos pertencentes ao espectro autistico.

\section{CONCLUSÃO}

Há alterações quantitativas e qualitativas no reconto de histórias em crianças diagnosticadas como autistas ou com Síndrome de Asperger avaliadas neste estudo. 


\section{ABSTRACT}

Purpose: to check the capacity of story retelling in children with PDD. Methods: the sample consisted of four verbal boys, 5 to 10-year old, diagnosed in multidisciplinary team with PDD and attended in the Department of Speech Therapy of the Federal University of São Paulo. For analyzing the linguistic performance, we used the history "The crow and the doves", proposed by Golden, reviewed / adapted by Antunes et. al. This story should be individually reproduced by each child after researcher's narrative. The recount was analyzed in a quantitative way, through the number of reproduced units (14 in the total) correctly by each child. And in a qualitative way, through the observation of the recall of the central themes and presence of distortions. Results: we verified that the number of reproduced items was 7 in average. However, it is interesting to note that nor all units considered central themes had been retold. None of the children made repetition and inversion. Conclusion: the results suggest that textual memory ability evidenced by story reproduction is not enough to assure comprehension, because some items are absent in thecentral themes retold by the subjects.

KEYWORDS: Autistic Disorder; Asperger Syndrome; Memory; Comprehension

\section{REFERÊNCIAS}

1. Organização Mundial da Saúde. A classificação CID-10 de desordens mentais e de comportamento. Genebra: Organização Mundial da Saúde; 1998. 2. Associação de Psiquiatria Americana. Manual diagnóstico e estatístico de transtornos mentais (DSM-IV). São Paulo: Artes Médicas; 2002.

3. Keith R. Central auditory tests. In: Lass NL, Mc Reynolds L, Northern J, Yoder D. Speech, language and hearing: hearing disorders. vol. 3. Philadelphia: WB Sauders; 1982.

4. Bowler DM, Gardiner JM, Berthollier N. Source memory in adolescents and adults with Asperger's syndrome. J Autism Develop Disord. 2004; 34(5):533-42.

5. Diehl JJ, Bennetto L, Young EC. Story recall and narrative coherence of high-functioning children with autism spectrum disorders. J Abnormal Child Psychol. 2006; 34(1):87-102.

6. Wang AT, Lee SS, Sigman M, Dapretto M. Neural basis of irony comprehension in children with autism: the role of prosody and context. Brain. 2006; 129(4):932-43.

7. Scheuer $\mathrm{Cl}$, Andrade RV. Teorias cognitivas e autism. In: Assumpção Junior FB, Kuczynski E. Autismo infantil: novas tendências e perspectivas. São Paulo: Atheneu; 2007. p. 81-90.

\section{DOI: 10.1590/S1516-18462009005000021}

RECEBIDO EM: 11/12/2007

ACEITO EM: 26/05/2008

Endereço para correspondência:

Juliana Onofre de Lira

Rua Euclides Pacheco, 803 ap. 1310

São Paulo - SP

CEP: 03321-000

E-mail: julira31@ terra.com.br.
8. Mason RA, Willians DL, Kana RK, Minshew N, Just MA. Theory of mind disruption and recruitment of the right hemisphere during narrative comprehension in autism. Neuropsychol. 2008; 46(1):269-80.

9. Eviatar Z, Marcel AJ. Brain correlates of discourse processing: an fMRI investigation of irony and conventional metaphor comprehension. Neuropsychol. 2006; 44(12):2348-59.

10. Harris CJ, Chabris CF, Clark J, Urban T, Aharon I, Steele $S$, et al. Brain activation during semantic processing in autism spectrum disorders via functional magnetic resonance imaging. Brain Cognition. 2006; 61(1):54-68.

11.Golden CJ. Luria Nebraska neuropsychological battery: children's revision. New York. Administration and Scoring Booklet; 1987.

12. Moraes Z, Chiari BM, Perissinoto J. Estilos de linguagem como facilitadores de memória. PróFono. 2001; 13(1):54-61.

13. Mandler JM, Johnson NS. Remembrance of things parced: story structure and recall. Cognitive Psychol. 1977; 9:111-5.

14. Fisher N, Happe F, Dunn J. The relationship between vocabulary, grammar, and false belief task performance inchildren withautisticspectrum disorders and children with moderate learning difficulties. J Child Psychol Psychiatr. 2005; 46(4):409-19.

15. Klin A. Autismo e Síndrome de Asperger: uma visão geral. Rev Bras Psiquiatr. 2006; 28(Supl1):13-8.

16. Perissinoto J. Diagnóstico de linguagem em crianças com Transtornos do Espectro Autistico. In: Ferreira LP, Befi Lopes DM, Limongi SCO, organizadores. Tratado de fonoaudiologia. São Paulo: Roca; 2004. p. 933-40.

17. Tamanaha AC, PerissinotoJ, ChiariBM. Umabreve revisão histórica sobre a construção dos conceitos do Autismo Infantil e da Síndrome de Asperger. Rev Soc Bras Fonoaudiol. 2008; 13(3):296-9. 